\title{
Option pricing in a hidden Markov model of the short rate with application to risky debt evaluation
}

\author{
Alessandro Ramponi ${ }^{\star}$ and Sergio Scarlatti \\ School of Economics, University of Rome - Tor Vergata, \\ via Columbia, 2 - 00133 Roma, Italy \\ Fax: +39062040219 Email: ramponi@economia.uniroma2.it \\ Email: scarlatti@economia.uniroma2.it \\ *Corresponding author
}

\begin{abstract}
Following the path initiated by Merton (1973), we study the option pricing problem in an economy with stochastic interest rates. We model the short rate dynamic by a diffusion process whose parameters are modulated by an underlying Markov process with jumps, as in Landen (2000). By exploiting the change of numeraire technique we obtain, under some assumption, a simple and easy to use call pricing formula which we then apply to the evaluation of risky debts so enlarging the flexibility of previous results obtained by Shimko et al. (1993). We also provide a detailed numerical study of call prices and credit spreads for a straightforward but interesting extension of the Vasicek dynamic included in our model.
\end{abstract}

Keywords: hidden Markov models; option pricing; regime switching models; risky debts; semi-affine term structure; T-forward measures.

Reference to this paper should be made as follows: Ramponi, A. and Scarlatti, S. (2009) 'Option pricing in a hidden Markov model of the short rate with application to risky debt evaluation', Int. J. Risk Assessment and Management, Vol. 11, Nos. 1/2, pp.88-103.

Biographical notes: A. Ramponi, $\mathrm{PhD}$, is assistant professor at the School of Economics, University of Rome 'Tor-Vergata'. His current research focuses on mathematical methods for financial markets and, in particular, on option pricing and term structure estimation and modelling. In the past he has also worked on Monte Carlo Markov Chain methods and their applications.

S. Scarlatti, PhD, is professor at the School of Economics, University of Rome 'Tor-Vergata'. His current research activities are in derivatives pricing under stochastic volatility, game theory and decisions. In the past he has also worked on simulation methods and pattern recognition problems.

\section{Introduction}

There is a growing interest in market models where the assets or the short-term interest rate dynamics exhibit shifts between different regimes (Ang and Bekaert, 2002; Bansal and Zhou, 2002; Buffington and Elliott, 2002; Dai and Singleton, 2003; Dai et al., 2005; Naik and Lee, 1997). Empirical evidence for the regime-switching behaviour of an economy was first reported by Hamilton (1989). In recent times, a 
number of attempts have been made to explain the corresponding dynamic processes by using hidden Markov models. This paper deals with a model for the short-term interest rate which has already been proposed and studied in the literature by Hansen and Poulsen (2000), Landen (2000) and Wu and Zeng (2004). However, differently from those who concentrate their work on bond pricing, we focus our study on option pricing. We are not the first to analyse the implications of a regime-switching effect on the prices of options, however, to our best knowledge, this has been done up to now only for the case of an underlying asset dynamic which directly incorporates a regime-switching term. A closer approach is considered in Elliott et al. (2005) but without taking into account a dynamic model for the term structure. In our work, assets follow a diffusive behaviour while discounting factors can display sudden jumps due to a shift of the short rate from one regime to another. To cope with this situation, we employ the well-known technique of change of numeraire. By so doing, we can build a forward measure and then, under quite a general hypothesis, we can provide a rather simple formula for pricing European call options. This formula has some nice features. Firstly, it is a self-evident and handy generalisation of Merton's formula. Secondly, even if it cannot be fully solved in closed form, it only requires a straightforward Monte Carlo procedure in order to estimate the values of the two (not explicitly solvable) expectations appearing within. We also provide a first natural application of this finding, namely to the evaluation of risky debts according to Merton's structural approach. Our credit spreads reduce to the credit spreads of Shimko et al. (1993) in case there are no jumps and the short rate follows a Vasiceck dynamic.

The paper is organised as follows: in Section 2 the term-structure model is presented. As in Landen (2000), we assume that all risk sources have already been priced by the market by selecting some martingale measure. In Section 3 we enlarge the set of tradeable assets to include a risky one and discuss conditions for the existence of forward measures as a tool for pricing contingent claims written on the risky asset. In Section 4, we give the result on the pricing of a European call and apply it to credit risk. We also discuss with full analytical details and the help of numerical simulations the case of a short-term interest rate which can revert only between two different levels, namely there are just two possible regime switchings in the economy.

\section{The term-structure model}

In this section, we shall introduce a model for the short-term interest rate which incorporates a regime-switching behaviour taking the works by Hansen and Poulsen (2000) and Landen (2000) as basic references. We start considering a financial market model living on a stochastic basis $\left(\Omega, \mathcal{F}, \mathcal{F}_{t}, Q\right)$ which is assumed to carry a one-dimensional standard Wiener process $W^{(r)}$ as well as a continuous-time and discrete-space Markov chain $X$ which we consider to be fully observable (for the partially observable case we refer to Landen (2000)). The main assets to be considered on the market are zero-coupon bonds with different maturities. For each maturity $T \geq 0 p(t, T)$ will denote the time $t$ price of a bond maturing at time $T$. The process $\{p(t, T): 0 \leq t \leq T\}$ is an optional stochastic process. We assume that there exists a 
frictionless market for $T$-bonds for every $T>0$. We define the instantaneous forward rate at $T$ contracted at time $t$ by $f(t, T)=-\partial \log p(t, T) / \partial T$ and the short rate as $r(t)=$ $f(t, t)$. The money market account process is defined by $B(t)=\exp \left(-\int_{0}^{t} r(s) d s\right)$. Lastly, we assume that the market is free of arbitrage, i.e. $Q$ is a martingale measure for the discounted bond price processes. The following system of stochastic differential equations defines our model for the short rate dynamic under $Q$ :

$$
\begin{aligned}
d r(t) & =\kappa(g(X(t))-r(t)) d t+\sigma(X(t)) d W^{(r)}(t), \\
d X(t) & =\int_{E} \delta(z) \mu(d t, d z)
\end{aligned}
$$

where $\mu(d t, d z)$ is a marked point process with finite mark space $(E, \mathcal{E}), E=\{z=(i, j)$ : $i \neq j, i, j \in\{1,2, \ldots N\}\}$ and $\mathcal{E}=2^{E}$; moreover $g(\cdot)$ and $\sigma(\cdot)>0$ are given functions, $\kappa>0$ is a constant and $\delta(z)=j-i$. The $Q$-compensator of $\mu(d t, d z)$ is given by the random measure $\nu(\omega ; d t, d z)=\lambda(t, X(t-, \omega), d z) d t$, where

$$
\lambda(t, X(t-, \omega), d z)=\sum_{i \neq j} h_{i j} 1_{(X(t-, \omega)=i)} \epsilon_{(i, j)}(d z)
$$

$\epsilon_{(i, j)}(d z)$ being the Dirac measure. In the previous formula, the numbers $h_{i j}$ are positive and such that $\sum_{j \neq i, j=1}^{N} h_{i j}=-h_{i i}$, for $i=1, \ldots, N$. In other terms, the modulating process $X(t)$ is a continuous time Markov chain with finite space $\{1,2, \ldots, N\}$ and intensity matrix $H=\left(h_{i j}\right)$. We remark that for $\sigma(x)=\sigma>0$ this model has been studied in Hansen and Poulsen (2000) and Landen (2000). Notice that the choice $g(x)=\theta$, with $\theta>0$, reproduces the classical Vasicek dynamic for the short rate (Vasicek, 1977). For all $T>t$ let

$$
p(t, T) \equiv F(t, T, r, x) \equiv \mathbf{E}^{Q}\left[e^{-\int_{t}^{T} r(s) d s} \mid r(t)=r, X(t)=x\right]
$$

define the underlying term-structure. It must verify the following integro-parabolic equation

$$
\partial_{t} F+k(g(x)-r) \partial_{r} F+\frac{\sigma^{2}(x)}{2} \partial_{r r}^{2} F+\int_{E} \Delta_{z} F \lambda(t, x, d z)=r F
$$

with terminal condition $F(T, T, r, x)=1$. In Equation (4) we have used the shorthand notation $\Delta_{z} F(t, T, r, x) \equiv F(t, T, r, x+\delta(z))-F(t, T, r, x)$.

By choosing $\log F(t, T, r, x)$ of the form $A(t, T, x)-B(t, T) r$ and substituting it directly into Equation (4), it is readily seen that we obtain a solution of the above equation whenever there are functions $A(t, T, x)$ and $B(t, T)$ which solve the integro-differential system:

$$
\begin{gathered}
\partial_{t} B-k B+1=0 \\
\partial_{t} A-k g(x) B+\frac{1}{2}(\sigma(x) B)^{2}+\int_{E}\left[e^{\Delta_{z} A}-1\right] \lambda(t, x, d z)=0
\end{gathered}
$$

with $A(T, T, x)=B(T, T)=0$. Term structures of the type $F(t, r, x, T)=$ $e^{A(t, T, x)-B(t, T, x) r}$ are termed semi-affine in Landen (2000). In summary, the bond market under consideration displays a semi-affine term structure with 


$$
B(t, T, x)=B(t, T)=\frac{1}{\kappa}\left(1-e^{-\kappa(T-t)}\right),
$$

provided Equation (6) admits finite solutions $A(t, T, x)$.

It is useful to notice that every function $F(x)$ having its support on $E$ can always be written as $F(x)=\sum_{i=1}^{N} F(i) 1_{(x=i)}$. Therefore, by means of the explicit expression of the compensator, Equation (6) for $A$ is equivalent to the following system of $N$ coupled equations

$$
\left\{\begin{array}{l}
\partial_{t} A_{1}-\kappa g_{1} B+\frac{1}{2}\left(\sigma_{1} B\right)^{2}+\sum_{j=1}^{N} h_{1 j} e^{A_{j}(t, T)-A_{1}(t, T)}=0 \\
\vdots \\
\partial_{t} A_{N}-\kappa g_{N} B+\frac{1}{2}\left(\sigma_{N} B\right)^{2}+\sum_{j=1}^{N} h_{N j} e^{A_{j}(t, T)-A_{N}(t, T)}=0
\end{array}\right.
$$

where $A_{i}(t, T)=A(t, T, i), g_{i}=g(i), \sigma_{i}=\sigma(i)$ and $A_{i}(T, T)=0$ for $i=1, \ldots, N$.

For $N=2$ and $\sigma_{1}=\sigma_{2}$ Landen provides explicit solutions for the functions $A_{1}, A_{2}$ in terms of Whittaker functions (see, for example, Abramowitz and Stegun (1972)).

The next proposition describes the stochastic differential equation (SDE) which must be satisfied by the T-bond price $p(t, T)$ under the short rate model dynamic in Equation (1).

Proposition 2.1. Suppose $r(t)$ and $X(t)$ solve the SDEs in Equation (1), then $p(t, T)$ solves

$$
\begin{aligned}
\frac{d p(t, T)}{p(t-, T)}= & \eta(t, T, r(t), X(t-)) d t-\sigma(X(t-)) B(t, T) d W^{(r)}(t) \\
& +\int_{E}\left[e^{\Delta_{z} A(t, T, X(t-))}-1\right] \mu(d t, d z)
\end{aligned}
$$

with

$$
\eta(t, T, r, x)=r-\int_{E}\left[e^{\Delta_{z} A(t, T, x)}-1\right] \lambda(t, x, d z)
$$

Proof. The generalised version of Ito's formula applied to a sufficiently smooth function $H(t, T, r, x)$ gives

$$
\begin{aligned}
d H= & \left(\partial_{t} H+\kappa(g(x)-r) \partial_{r} H+\frac{1}{2} \sigma(x)^{2} \partial_{r r}^{2} H\right) d t \\
& +\sigma(x) \partial_{r} H d W^{(r)}(t)+\int_{E} \Delta_{z} H \mu(d t, d z)
\end{aligned}
$$

where $\Delta_{z} H \equiv \Delta_{z} H(t, T, r, x) \equiv H(t, T, r, x+\delta(z))-H(t, T, r, x)$. We apply the previous formula to the function $H(t, T, r, x)=e^{A(t, T, x)-r B(t, T)}$. Noticing that the semi-affine structure implies

$$
\Delta_{z} H=H\left(e^{\Delta_{z} A}-1\right)
$$

and taking into account the relations

$$
\partial_{t} H=\left(\partial_{t} A-r \partial_{t} B\right) H, \quad \partial_{r} H=-B H, \quad \partial_{r r}^{2} H=B^{2} H,
$$

we obtain 


$$
\begin{aligned}
\eta(t, T, r, x) & =\left(\partial_{t} A-r \partial_{t} B\right)-\kappa(g(x)-r) B+\frac{1}{2}(\sigma(x) B)^{2} \\
& =r-\int_{E}\left[e^{\Delta_{z} A(t, T, x)}-1\right] \lambda(t, x, d z)
\end{aligned}
$$

where we have used Equation (6). This ends the proof.

Therefore, while the short rate process has continuous paths, the prices of zero coupon bonds can display jumps.

\section{Forward measures and the pricing of contingent claims}

A standard useful trick for pricing contingent claims in an economy with stochastic interest rates consists in the use of the so called $T$-forward measure introduced by Geman et al. (1995) and Jamshidian (1989) (see also Bjork, 2000). In this section we shall be mainly interested in pricing European options on a single asset. We assume:

- the asset is traded on the market

- under the measure $Q$ the asset price process is given by the solution of the SDE

$$
d S(t)=S(t)\left[r(t) d t+\xi d W^{(s)}(t)\right]
$$

with $\xi>0$.

The one-dimensional Wiener process $W^{(s)}$ lives on the same stochastic basis $\left(\Omega, \mathcal{F}, \mathcal{F}_{t}, Q\right)$ which carries the Wiener process $W^{(r)}$, moreover the two processes are correlated with correlation coefficient $\rho \in[-1,1]$. This allows for the representation

$$
\begin{aligned}
& W^{(r)}(t)=W^{(1)}(t) \\
& W^{(s)}(t)=\rho W^{(1)}(t)+\sqrt{1-\rho^{2}} W^{(2)}(t)
\end{aligned}
$$

where $W^{Q}(t)=\left(W^{(1)}(t), W^{(2)}(t)\right)$ is a standard two-dimensional Wiener process.

The $T$-forward measure is now defined to be a probability measure $Q^{T}$ (absolutely continuous with respect to $Q$ ) under which the process $u \rightarrow Z_{t}^{T}(u)$, $t \leq u \leq T$, defined by

$$
Z_{t}^{T}(u)=\frac{S(u)}{p(u, T)}
$$

is a martingale with respect to the natural filtration $\mathcal{G}=\left\{\mathcal{G}_{t, u}\right\}_{u \geq t}$ generated by the processes $W^{Q}(\cdot)$ and $\mu(\cdot, \cdot)$, i.e.

$$
\mathcal{G}_{t, u}=\mathcal{H}_{t, u} \vee \mathcal{K}_{t, u} \vee \mathcal{N}
$$

where $\mathcal{H}_{t, u}=\sigma\left\{W^{Q}(s), t \leq s \leq u\right\}, \mathcal{K}_{t, u}=\sigma\{\mu([t, s] \times A), s \leq u, A \in \mathcal{E}\}$ and $\mathcal{N}$ is the collection of $Q$-null sets from $\mathcal{F}$.

Let $\Pi(t, T)$ denote the price at time $t$ of an option with maturity $T$ and terminal pay-off $\Phi(S(T))$ having finite expectation with respect to $Q^{T}$. Since $p(T, T)=1$ by the martingale property this implies

$$
\Pi(t, T)=p(t, T) \mathbf{E}^{T}\left[\Phi\left(Z_{t}^{T}(T)\right) \mid \mathcal{G}_{0, t}\right]
$$


where $\mathbf{E}^{T}$ is the expectation with respect to the $Q^{T}$ measure.

In order to show the existence of a measure $Q^{T}$ and to identify the $Q^{T}$-dynamic of the process $Z_{r}^{T}(t)$ we first compute the dynamic of $Z_{r}^{T}(t)$ under the measure $Q$. This can be easily obtained by a straightforward use of the Ito formula for semi-martingales applied to the function $G(s, p)=\frac{s}{p}$ evaluated at $(S(t), p(t, T))$ (see, for example, Protter (1990)), which gives:

$$
\begin{aligned}
\frac{d Z_{u}^{T}(t)}{Z_{u}^{T}(t-)}= & m(t, T, X(t-)) d t+\omega(t, T, X(t-)) \cdot d W^{Q}(t) \\
& +\int_{E}\left(e^{-\Delta_{z} A(t, T, X(t-))}-1\right) \mu(d t, d z)
\end{aligned}
$$

where

$$
\begin{aligned}
& m(t, T, x)=\sigma^{2}(x) B^{2}(t, T)+\xi \rho \sigma(x) B(t, T)+\int_{E}\left[e^{\Delta_{z} A(t, T, X(t-))}-1\right] \lambda(t, x, d z) \\
& \omega(t, T, x)=\left(\xi \rho+\sigma(x) B(t, T), \xi \sqrt{1-\rho^{2}}\right) .
\end{aligned}
$$

In the calculations of Equation (15) we have used the key identity

$$
\Delta_{p} G=G\left(e^{-\Delta_{z} A}-1\right)
$$

which measures the variation of $G$ due to a term-structure jump.

The following proposition exploits a suitable version of Girsanov's theorem for marked point processes (see Bjork et al. (1997) and Brémaud (1981), Chapter VIII) to find a driftless dynamic for $Z_{u}^{T}(t)$.

Theorem 3.1. Suppose the following hypothesis hold:

Hypothesis 1. There is a predictable process $\Gamma(t)=\left(\Gamma_{1}(t), \Gamma_{2}(t)\right) \in \mathbb{R}^{2}$ and a measurable (with respect to the predictable $\sigma$-algebra) strictly positive function $\Phi(t, z)$ such that

$$
\begin{aligned}
m(t, T, X(t-)) & +\omega(t, T, X(t-)) \cdot \Gamma(t) \\
& +\int_{E}\left(e^{-\Delta A_{z}(t, T, X(t-))}-1\right) \Phi(t, z) \lambda(t, X(t-), d z)=0
\end{aligned}
$$

is satisfied $d Q d t$ almost everywhere (a.e.) on $\Omega \times[0, T]$ and

$$
\int_{0}^{T}\|\Gamma(s)\|^{2} d s<\infty, \quad \int_{0}^{T} \int_{E} \Phi(s, z) \lambda(s, X(s-), d z) d s<\infty
$$

$d Q$ a.e.

Hypothesis 2. The process $L(t)$ defined by

$$
\begin{aligned}
\log L(t)= & \int_{0}^{t} \Gamma(s) \cdot d W^{Q}(s)-\frac{1}{2} \int_{0}^{T}\|\Gamma(s)\|^{2} d s \\
& +\int_{0}^{t} \int_{E} \log \Phi(s, z) \mu(d s, d z)+\int_{0}^{t} \int_{E}(1-\Phi(s, z)) \lambda(s, X(s-), d z) d s
\end{aligned}
$$


verifies the condition

$$
\mathbf{E}^{Q}(L(T))=1 .
$$

Then there exists a probability measure $Q^{T}$ equivalent to $Q$ on $\mathcal{G}_{0, T}$ with density $\frac{d Q^{T}}{d Q}=L(T)$ such that:

- the two-dimensional process

$$
\tilde{W}(t)=W^{Q}(t)-\int_{0}^{t} \Gamma(s) d s
$$

is a standard $Q^{T}$-Wiener process

- the point process $\mu$ has $Q^{T}$-intensity given by

$$
\lambda^{T}(t, x, d z)=\Phi(t, z) \lambda(t, x, d z) ;
$$

- let $\bar{\mu}^{T}(d t, d z)=\mu(d t, d z)-\lambda^{T}(t, X(t-), d z) d t$ be the $Q^{T}$-compensated jump process then, under $Q^{T}$, the process $Z_{u}^{T}(t)$ solves the following $\mathrm{SDE}$

$$
\frac{d Z_{u}^{T}(t)}{Z_{u}^{T}(t-)}=\omega(t, T, X(t-)) \cdot d \tilde{W}(t)+\int_{E}\left(e^{-\Delta_{z} A(t, T, X(t-))}-1\right) \bar{\mu}^{T}(d t, d z)
$$

and, therefore, is a martingale.

Proof. The proof is straightforward. By Hypotheses 1 and 2 (Brémaud, 1981) there exists a probability measure $Q^{T}$ on $\mathcal{G}_{0, T}$ equivalent to $Q$ with density $L(T)$ such that $\tilde{W}(t)$ is a standard $Q^{T}$-Wiener process and the point process $\mu$ has $Q^{T}$ intensity given by

$$
\lambda^{T}(t, x, d z)=\Phi(t, z) \lambda(t, x, d z) .
$$

Moreover, under $Q^{T}$ we clearly have

$$
\begin{aligned}
\frac{d Z_{u}^{T}(t)}{Z_{u}^{T}(t-)}= & (m(t, T, X(t-))+\omega(t, T, X(t-)) \cdot \Gamma(t) \\
& \left.+\int_{E}\left(e^{-\Delta A_{z}(t, T, X(t-))}-1\right) \lambda^{T}(t, X(t-), d z)\right) d t+\omega(t, T) d \tilde{W}(t) \\
& +\int_{E}\left(e^{-\Delta A_{z}(t, T, X(t-))}-1\right)\left(\mu(d t, d z)-\lambda^{T}(t, X(t-), d z) d t\right)
\end{aligned}
$$

where we have compensated $\mu$. Finally we use Equation (16) to cancel the drift in the last equation. This ends the proof.

Let us now discuss more closely Hypotheses 1 and 2. We see that Hypothesis 1 requires to exhibit solutions $\left(\Gamma_{1}(t), \Gamma_{2}(t)\right)$ and $\Phi(t, z)$ of Equation (16). Furthermore, they must verify some integrability conditions but these are always satisfied in our model, $E$ being a finite space. We notice that Equation (16) can be explicitly rewritten in the form

$$
\begin{aligned}
\sigma^{2}(X(t-)) B(t, T)^{2} & +\rho \xi \sigma(X(t-)) B(t, T)+\left(\rho \xi+\sigma(X(t-)) B(t, T) \Gamma_{1}(t)\right) \\
& +\xi \sqrt{1-\rho^{2}} \Gamma_{2}(t)+\int_{E}\left(e^{-\Delta A_{z}(t, T, X(t-))}-1\right) \Phi(t, z) \lambda(t, X(t-), d z) \\
& +\int_{E}\left(e_{-}^{\Delta A_{z}(t, T, X(t-))}-1\right) \lambda(t, X(t-), d z)=0 .
\end{aligned}
$$


There are plenty of solutions $(\Gamma, \Phi)$ to this equation, each one giving rise to a $Q^{T}$ forward measure. However, these $Q^{T}$-forward measures all assign the same value to the option by means of Equation (14). Henceforth, we can just select one of them in the most convenient way. A natural one is to split Equation (20) into the system:

$$
\begin{aligned}
& \sigma^{2}(X(t-)) B(t, T)^{2}+\rho \xi \sigma(X(t-)) B(t, T)+\left(\rho \xi+\sigma(X(t-)) B(t, T) \Gamma_{1}(t)\right) \\
& \quad+\xi \sqrt{1-\rho^{2}} \Gamma_{2}(t)=0 \\
& \int_{E}\left(e^{-\Delta A_{z}(t, T, X(t-))}-1\right) \Phi(t, z) \lambda(t, X(t-), d z) \\
& \quad+\int_{E}\left(e^{\Delta A_{z}(t, T, X(t-))}-1\right) \lambda(t, X(t-), d z)=0
\end{aligned}
$$

therefore separating the diffusive and jump parts. We begin discussing the first equation. There are two cases: $\rho= \pm 1$ and $\rho \neq \pm 1$.

Case $\rho= \pm 1$ : the equation is solved by

$$
\Gamma_{1}(t)=-\frac{\rho \xi \sigma(X(t-))+\sigma^{2}(X(t-)) B(t, T)}{\rho \xi+\sigma(X(t-))} .
$$

Case $\rho \neq \pm 1$ : we solve the equation by choosing

$$
\begin{aligned}
& \Gamma_{1}(t)=-\frac{\sigma^{2}(X(t-)) B(t, T)^{2}}{\rho \xi+\sigma(X(t-)) B(t, T)} \\
& \Gamma_{2}(t)=-\frac{\rho}{\sqrt{1-\rho^{2}}} \sigma(X(t-)) B(t, T) .
\end{aligned}
$$

In particular, for $\rho=0$ we have $\Gamma_{1}(t)=-\sigma(X(t-)) B(t, T)$ and $\Gamma_{2}(t) \equiv 0$.

We now analyse the second equation. Notice that in case there are no jumps $\Delta A_{z} \equiv 0$ so it reduces to a trivial identity. Next we focus on the special case $N=2$. By using Equation (2), writing $\Phi(t, z)$ as $\Phi_{i j}(t)$ for $z=(i, j) i \neq j$ and setting

$$
G_{i j}(t, T)=A_{j}(t, T)-A_{i}(t, T),
$$

we see that the equation is equivalent to the two conditions

$$
\begin{aligned}
& h_{12} \Phi_{12}(t)\left(e^{-G_{12}(t, T)}-1\right)+h_{12}\left(e^{G_{12}(t, T)}-1\right)=0 \\
& h_{21} \Phi_{21}(t)\left(e^{-G_{21}(t, T)}-1\right)+h_{21}\left(e^{G_{21}(t, T)}-1\right)=0
\end{aligned}
$$

whose explicit solutions are given by

$$
\begin{aligned}
& \Phi_{12}(t)=e^{G_{12}(t, T)} \\
& \Phi_{21}(t)=e^{G_{21}(t, T)},
\end{aligned}
$$

which are strictly positive. Permuting the indices, we pass from one to the other. For $N \geq 3$, using Equation (2), we can again reduce the initial equation to a linear system of $N$ equations in the $N(N-1)$-unknowns $\Phi_{i j}(t), i, j=1, \ldots, N$ with $i \neq j$. We have an underdetermined linear system from which we must select out some (positive) solutions. Since we shall be most interested in our applications to the $N=2$ case we 
do not expand the analysis of this point furthermore. Finally, we notice that the requirement Hypothesis 2 is also satisfied. Indeed the usual exponential moments finiteness condition (see (3.9) in Brémaud (1981)) which implies Equation (18), it clearly holds in our case again by the finiteness of the space $E$.

\section{Valuation of corporate risky debts}

In this section we shall give an important application to corporate risky debt valuation of the results obtained in Section 3. We shall take Merton's structural approach (Merton, 1974) as our guideline, see Duffie and Singleton (2003) for an overview. Generalisations of Merton results to the case of stochastic interest have already appeared, for example, Shimko et al. (1993) covered the case of a short rate Vasiceck-dynamic (more recently Wong and Kwok (2003) allowed for jumps in the asset dynamic). Merton's approach becomes especially powerful when there is the possibility to determine, in the chosen market model, the price of a European call option in relatively simple form. To this aim we shall denote by $X^{t, i}(s), s \geq t$, the Markov chain starting in the state $i$ at time $t$, by $p_{i}(t, T)$ the related term-structure and by $Z_{t, i}^{T}(s)$ the corresponding solution, under the measure $Q^{T}$, of the stochastic differential equation (Equation (19)) for $s \geq t$ given $S(t)=S$. The logarithm of $Z_{t, i}^{T}(s)$ evaluated at time $s=T$ has the form (see, for example, Runggaldier (2002)):

$$
\begin{aligned}
\log Z_{t, i}^{T}(T)= & \log Z_{t, i}^{T}(t)-\int_{t}^{T}\left(\int_{E}\left(e^{-\Delta_{z} A}-1\right) \lambda^{T}\left(s, X^{t, i}(s-), d z\right)\right. \\
& \left.+\frac{1}{2}\left\|\omega\left(s, T, X^{t, i}(s-)\right)\right\|^{2}\right) d s+\int_{t}^{T} \omega\left(s, T, X^{t, i}(s-)\right) \cdot d \tilde{W}(s) \\
& -\int_{t}^{T} \int_{E} \Delta_{z} A \mu(d s, d z) .
\end{aligned}
$$

We shortly rewrite it as

$$
\log Z_{t, i}^{T}(T)=N_{i}(t, T)+Y_{i}(t, T)
$$

where

$$
\begin{aligned}
& N_{i}(t, T)=\log \left(\frac{S}{p_{i}(t, T)}\right)-\frac{1}{2} V_{i}^{2}(t, T)+\int_{t}^{T} \omega\left(s, T, X^{t, i}(s-)\right) \cdot d \tilde{W}(s) \\
& Y_{i}(t, T)=-\int_{t}^{T} \int_{E}\left(e^{-\Delta_{z} A}-1\right) \lambda^{T}\left(s, X^{t, i}(s-), d z\right) d s-\int_{t}^{T} \int_{E} \Delta_{z} A \mu(d s, d z),
\end{aligned}
$$

and

$$
V_{i}(t, T)=\left[\int_{t}^{T}\left\|\omega\left(s, T, X^{t, i}(s-)\right)\right\|^{2} d s\right]^{1 / 2} .
$$

Let $\mathcal{N}(\cdot)$ denote the cumulative distribution of a standard normal, the following result holds: 
Proposition 4.1. For any pair of variables $(\chi, \Omega)$ define the functions

$$
\begin{aligned}
& d_{2, i}(\chi, \Omega)=\left[\log \left(\frac{S}{\chi p_{i}(t, T)}\right)-\frac{1}{2} \Omega^{2}\right] / \Omega \\
& d_{1, i}(\chi, \Omega)=d_{2, i}(\chi, \Omega)+\Omega,
\end{aligned}
$$

then the price at time $t$ of a European call option with maturity $T$ and strike price $K$ is given by

$$
c_{i}(t, T, S, K)=S \alpha_{i}-K p_{i}(t, T) \beta_{i}
$$

where

$$
\alpha_{i}=\mathbf{E}^{T}\left[e^{Y_{i}(t, T)} \mathcal{N}\left[d_{1, i}\left(K e^{-Y_{i}(t, T)}, V_{i}(t, T)\right)\right]\right]
$$

and

$$
\beta_{i}=\mathbf{E}^{T}\left[\mathcal{N}\left[d_{2, i}\left(K e^{Y_{i}(t, T)}, V_{i}(t, T)\right)\right]\right]
$$

Proof. We have

$$
\begin{aligned}
c_{i}(t, T, S, K) & =p_{i}(t, T) \mathbf{E}^{T}\left[\left(Z_{t, i}^{T}(T)-K\right)^{+} \mid \mathcal{G}_{0, t}\right] \\
& =p_{i}(t, T) \mathbf{E}^{T}\left[\left(e^{N_{i}(t, T)+Y_{i}(t, T)}-K\right)^{+}\right] \\
& \left.=p_{i}(t, T) \mathbf{E}^{T}\left[\mathbf{E}^{T}\left(e^{N_{i}(t, T)+Y_{i}(t, T)}-K\right)^{+} \mid \mathcal{K}_{t, T}\right)\right] \\
& \left.=p_{i}(t, T) \mathbf{E}^{T}\left[e^{Y_{i}(t, T)} \mathbf{E}^{T}\left(e^{N_{i}(t, T)}-e^{-Y_{i}(t, T)} K\right)^{+} \mid \mathcal{K}_{t, T}\right)\right] \\
& \left.=\mathbf{E}^{T}\left[e^{Y_{i}(t, T)}\left(p_{i}(t, T) \mathbf{E}^{T}\left(e^{N_{i}(t, T)}-e^{-Y_{i}(t, T)} K\right)^{+} \mid \mathcal{K}_{t, T}\right)\right)\right] .
\end{aligned}
$$

We notice now that, under $Q^{T}$,

$$
N_{i}(t, T) \mid \mathcal{K}_{t, T} \sim \mathcal{N}\left(\log \left(\frac{S}{p_{i}(t, T)}\right)-\frac{1}{2} V_{i}^{2}(t, T), V_{i}^{2}(t, T)\right) .
$$

Therefore we have

$$
\begin{aligned}
c_{i}(t, S, K, T)= & \mathbf{E}^{T}\left[e^{Y_{i}(t, T)} S \mathcal{N}\left(d_{1, i}\left(K e^{-Y_{i}(t, T)}, V_{i}(t, T)\right)\right)\right] \\
& \left.-K p_{i}(t, T) \mathbf{E}^{T}\left[\mathcal{N}\left(d_{2, i}\left(K e^{-Y_{i}(t, T)}, V_{i}(t, T)\right)\right)\right)\right] \\
= & S \alpha_{i}-K p_{i}(t, T) \beta_{i} .
\end{aligned}
$$

Notice that $\alpha_{i}$ and $\beta_{i}$ are always finite numbers since $\mathcal{N}(\cdot) \leq 1$ and $\mathbf{E}^{T}\left(e^{Y_{i}(t, T)}\right)<\infty$. This ends the proof.

Remark. Clearly if the market is such that $Y_{i}(t, T) \equiv 0$ a.e. then we fully recover Merton's formula (Merton, 1974). 
Suppose now the process $(S(u))_{u>t}$ represents the firm's asset value and, following Merton, assume that default can occur only when the firm cannot repay at time $T$ the promised principal, denoted by $F$. In case of default, bondholders take over the firm and shareholders get nothing. Under such circumstances the value of a corporate discount bond with maturity $T$ can be evaluated by the following formula:

$$
\begin{aligned}
D_{i}(t, T)= & p_{i}(t, T) \mathbf{E}^{T}\left[S(T)-(S(T)-F)^{+} \mid \mathcal{G}_{0, t}\right] \\
= & p_{i}(t, T) \mathbf{E}^{T}\left[Z_{t, i}^{T}(T) \mid \mathcal{G}_{0, t}\right]-p_{i}(t, T) \mathbf{E}^{T}\left[\left(Z_{t, i}^{T}(T)-F\right)^{+} \mid \mathcal{G}_{0, t}\right] \\
= & S(t)-c_{i}(t, T, S(t), F)=S(t)-S(t) \mathbf{E}^{T}\left[e^{Y_{i}(t, T)} \mathcal{N}\left(d_{1}\left(F e^{-Y_{i}(t, T)}\right)\right)\right] \\
& -F p_{i}(t, T) \mathbf{E}^{T}\left[\mathcal{N}\left(d_{2}\left(F e^{-Y_{i}(t, T)}\right)\right)\right],
\end{aligned}
$$

where the last equality comes from our previous result. Hence,

$$
\begin{aligned}
D_{i}(t, T)= & p_{i}(t, T)\left\{Z _ { t , i } ^ { T } ( t ) \left(1-\mathbf{E}^{T}\left[e^{Y_{i}(t, T)} \mathcal{N}\left(d_{1}\left(F e^{-Y_{i}(t, T)}\right)\right)\right]\right.\right. \\
& \left.+F \mathbf{E}^{T}\left[\mathcal{N}\left(d_{2}\left(F e^{-Y_{i}(t, T)}\right)\right)\right]\right\} .
\end{aligned}
$$

Therefore credit spreads have the following expression

$$
\begin{aligned}
& \delta_{i}(t, T)=-\frac{1}{T-t} \log \left(\frac{D_{i}(t, T)}{F p_{i}(t, T)}\right) \\
& =-\frac{1}{T-t} \log \left(\frac{Z_{t, i}^{T}(t)}{F}\left(1-\mathbf{E}^{T}\left[e^{Y_{i}(t, T)} \mathcal{N}\left(d_{1}\left(F e^{-Y_{i}(t, T)}\right)\right)\right]\right)+\mathbf{E}^{T}\left[\mathcal{N}\left(d_{2}\left(F e^{-Y_{i}(t, T)}\right)\right)\right]\right)(26) \\
& =-\frac{1}{T-t} \log \left(\frac{1}{k_{i}(t, T)}\left(1-\mathbf{E}^{T}\left[e^{Y_{i}(t, T)} \mathcal{N}\left(d_{1}\left(F e^{-Y_{i}(t, T)}\right)\right)\right]\right)+\mathbf{E}^{T}\left[\mathcal{N}\left(d_{2}\left(F e^{-Y_{i}(t, T)}\right)\right)\right]\right)
\end{aligned}
$$

$k_{i}(t, T)=\frac{F}{Z_{t, i}^{T}(t)}$ being the discounted debt to asset ratio or firm's leverage. We shall now discuss the behaviour of these credit spreads for $N=2$.

Case $N=2$. The presence of only two different levels is a special but already interesting framework. In particular, the bond pricing problem has been studied in detail in the papers by Hansen and Poulsen (2000) and Landen (2000) in case $h_{12}=h_{21}=h$ and $\sigma(1)=\sigma(2)=\sigma$. We shall assume these last conditions and discuss the corresponding option pricing problem. The solution essentially consists of a three-step procedure:

- Firstly the semi-affine structure must be explicitly solved (or numerically computed) under the martingale measure $Q$.

- Secondly, once the functions $A_{i}(t, T)$ and $B(t, T)$ are available, the Markov chain $\tilde{X}$ driven by the time-dependent intensity matrix

$$
\left(h_{i j}^{T}(s)\right)_{i, j=1,2}=\left(\begin{array}{cc}
-h \Phi_{12}(s) & h \Phi_{12}(s) \\
h \Phi_{21}(s) & -h \Phi_{21}(s)
\end{array}\right)
$$

$t \leq s \leq T$, needs to be taken into account for the evaluation of the weights $e^{\overline{Y_{i}}(t, T)}$ appearing in the pricing formula (Equation (23)), where the expectation is performed with respect to the measure $Q^{T}$. 
- Finally, the prices are computed using Monte Carlo averages of very simple functionals according to Equation (23), where for $i=1,2$

$$
\begin{aligned}
V_{i}(t, T) \equiv V(t, T)= & \left((T-t)\left(\xi^{2}+\frac{\sigma^{2}}{k^{2}}+\frac{2 \rho \xi \sigma}{k}\right)+\left(e^{-k(T-t)}-1\right)\left(\frac{2 \sigma^{2}}{k^{3}}+\frac{2 \rho \xi \sigma}{k^{2}}\right) .\right. \\
& \left.-\frac{\sigma^{2}}{2 k^{3}}\left(e^{-2 k(T-t)}-1\right)\right)^{1 / 2} \cdot
\end{aligned}
$$

In order to explain these steps in more detail it is convenient to fix some notations. The numbers $g_{1}$ and $g_{2}$ will represent the two possible levels of mean reversion for the short rate process. We take $g_{1}$ and $g_{2}$ positive with $\epsilon=g_{1}-g_{2} \geq 0$ being the size of their gap, i.e. $g_{1}$ is the 'high' level. For $i \in\{1,2\}$ we set

$$
G_{i j}(t, T)=A_{j}(t, T)-A_{i}(t, T),
$$

$\left(A_{j}(t, T)=A(t, T, j), j=1,2\right.$ as in Section 2). Clearly $\Delta A(t, T, i, z)=G_{i \hat{i}}(t, T), \hat{i}$ being the complementary state of $i$. The process $Y_{i}(t, T)$ can be explicitly written as:

$$
\begin{aligned}
Y_{i}(t, T)= & -\sum_{n=1}^{N_{T}}\left(1_{\tilde{X}^{t, i}\left(\tau_{n}-\right)=2}-1_{\tilde{X}^{t, i}\left(\tau_{n}-\right)=1}\right) G_{21}\left(\tau_{n}, T\right) \\
& -\int_{t}^{T}\left(h_{21}^{T}(s) 1_{\tilde{X}^{t, i}(s-)=2}\left(e^{-G_{21}(s, T)}-1\right)+h_{12}^{T}(s) 1_{\tilde{X}^{t, i}(s-)=1}\left(e^{-G_{12}(s, T)}-1\right)\right) d s
\end{aligned}
$$

where $N_{T}$ is the total number of times the chain changes its state in $(0, T]$ and the random times $t<\tau_{1}<\tau_{2} \ldots<\tau_{N_{T}} \leq T$ are the jumping (regime-switching) times.

We recall that for $N=2$ the system of Equation (7) reduces to

$$
\left\{\begin{array}{l}
\frac{\partial}{\partial t} A_{1}(t, T)-\kappa g_{1} B(t, T)+\frac{1}{2}(\sigma B(t, T))^{2}+h\left(e^{-G_{21}(t, T)}-1\right)=0 \\
\frac{\partial}{\partial t} A_{2}(t, T)-\kappa g_{2} B(t, T)+\frac{1}{2}(\sigma B(t, T))^{2}+h\left(e^{G_{21}(t, T)}-1\right)=0
\end{array}\right.
$$

with $A_{1}(T, T)=A_{2}(T, T)=0$ and $B(t, T)=(1-\exp (-\kappa(T-t))) / \kappa$. In particular, it follows that the function $G_{21}(t, T)$ verifies the following nonlinear ordinary differential equation (ODE):

$$
\left\{\begin{aligned}
\frac{\partial}{\partial t} G_{21}(t, T) & =k \epsilon B(t, T)+2 h \sinh \left(G_{21}(t, T)\right) \\
G_{21}(T, T) & =0
\end{aligned}\right.
$$

Remark. For $\epsilon=0$, i.e. $g_{1}=g_{2}=\theta$, the choice $G_{21}(t, T) \equiv 0$ clearly solves the equation and, as a consequence, $Y_{i}(t, T)=0$ with probability 1 . Moreover, $p(t, T)=p_{\theta}^{V}(t, T)$, i.e. $p(t, T)$ coincides with the Vasicek affine term structure having $\theta$ as mean reversion level. Henceforth the price of a call option is given by

$$
c(t, T ; K)=c^{V M}(t, T ; K)
$$

where

$$
c^{V M}(t, T ; K)=S(t) \mathcal{N}\left(d_{1}^{V}(K)\right)-K p_{\theta}^{V}(t, T) \mathcal{N}\left(d_{2}^{V}(K)\right)
$$


and

$$
\begin{aligned}
& d_{2}^{V}(K)=\left(\log \left(\frac{S(t)}{K p_{\theta}^{V}(t, T)}\right)-\frac{1}{2} V^{2}(t, T)\right) / V(t, T) \\
& d_{1}^{V}(K)=d_{2}^{V}(K)+V(t, T) .
\end{aligned}
$$

We recall that explicit solutions of Equation (29) are available in terms of Whittaker functions (Landen, 2000). By using such a representation, it can be easily seen that

where

$$
G_{12}(t, T)=\ln \frac{\zeta_{t}(t, T)}{h \zeta(t, T)}
$$

$$
\zeta(t, T)=e^{\gamma(\exp (k t))} w\left(2 C_{2} e^{k t}\right)
$$

and $w$ is defined by means of the Whittaker functions $M_{k, m}$ and $W_{k, m}$ (see, for example, Abramowitz and Stegun (1972)) as

$$
w(u)=D_{1} M_{l, m}(u)+D_{2} W_{l, m}(u)
$$

with $l=-C_{1}, m=\sqrt{1 / 4-C_{1}+C_{1}^{2}+(h / k)^{2}}$ and

$$
\gamma(s)=\frac{(k-\epsilon) \log (s)}{2 k}-\frac{s \epsilon}{2 k e^{k T}}+\frac{(k-\epsilon) T}{2}+\frac{\epsilon}{2 k} .
$$

The exact values of the constants $C_{1}, C_{2}$ and $D_{1}, D_{2}$ can be found in Landen (2000). However, we remark that, even if such a representation is available, its practical implementation is questionable and we have to resort to numerical methods for solving Equation (30) in order to get significant bond price curves, as in Landen (2000) also.

We now report on a set of numerical simulations performed within this model showing the behaviour of call prices (Equation (23)) and credit spreads (Equation (26)) for a selected set of parameters. In order to evaluate the expectations involved in our formulae, we must resort to Monte Carlo simulations of the processes $Y_{i}(t, T), i=1,2$.

Once the function $G_{21}(s, T), t \leq s \leq T$ is available for computation, e.g. by numerically solving Equation (30), independent samples of the variable $Y_{i}(t, T)$ are easily obtained by simulating independent paths of the two-state continuous time non-homogeneous Markov chain $\tilde{X}(s), t \leq s \leq T$ and then evaluating the corresponding sample $Y_{i}^{(m)}(t, T)$ through Equation (28), where a numerical integration method is needed. Since the matrix elements (Equation (27)) are bounded over the interval $[t, T]$ the previous simulation step can be efficiently realised by means of the thinning algorithm (see Glasserman and Merener (2004) for a recent application of this technique in financial mathematics). The expectations in Equation (23) can be, then, approximated by

$$
\mathbf{E}^{T}\left[e^{Y_{i}(t, T)} \mathcal{N}\left(d_{1}\left(K e^{-Y_{i}(t, T)}\right)\right)\right] \approx \frac{1}{M} \sum_{m=1}^{M} e^{Y_{i}^{(m)}(t, T)} \mathcal{N}\left(d_{1}\left(K e^{-Y_{i}^{(m)}(t, T)}\right)\right)
$$

and

$$
\mathbf{E}^{T}\left[\mathcal{N}\left(d_{2}\left(K e^{-Y_{i}(t, T)}\right)\right)\right] \approx \frac{1}{M} \sum_{m=1}^{M} \mathcal{N}\left(d_{2}\left(K e^{-Y_{i}^{(m)}(t, T)}\right)\right)
$$

for a sufficiently large $M$. 
Without loss of generalisation, in our experiments we set $t=0$. In Figure 1 , the results of some numerical simulations showing the impact of the model parameters $T$, $\epsilon, h$ and $\rho$ on the call prices are reported. The gap was incremented by fixing the lower level $g_{2}$ and varying the higher level as $g_{1}=g_{2}+\epsilon$. The other (fixed) parameters were chosen as: for the short rate dynamic, $\kappa=1$ and $\sigma^{(r)}=0.02$ and for the price process, $\sigma^{(s)}=0.2$. In our experiment, $M=20,000$ samples were sufficient to approximate the expectations within a $95 \%$ confidence interval of length about $10^{-3}$. The results obtained with the two-state regime switching model are compared with those obtained by using the Merton model. In all plots the '*' and dotted '*' curves are the call prices in our regime switching model with $X(0)=g_{1}$ and $X(0)=g_{2}$, respectively. The 'o' and dotted 'o' curves are the corresponding Merton prices. For this last model we used the Vasicek short rate term structure with mean level parameter $X(0)$ and, again, $\kappa=1, \sigma^{(r)}=0.02$ and $\sigma^{(s)}=0.2$. The initial short rate value $r(0)$ was chosen as $r(0)=\left(g_{1}+g_{2}\right) / 2$ for all plots unless the one where $\epsilon$ is varying - in that case it was fixed as $r(0)=0.07$. In all these experiments we set $S(0)=100$ and $K=100$.

Figure 1 Call prices as a function of the model parameters $T, \epsilon, h$ and $\rho$. For each plot the fixed parameters were chosen as $\kappa=1, \sigma^{(r)}=0.02, g_{2}=0.05, g_{1}=0.1, h=1$, $r(0)=\left(g_{1}+g_{2}\right) / 2, T=5, S(0)=100, K=100, \sigma^{(s)}=0.2, \rho=0.5$ (for colours see online version)
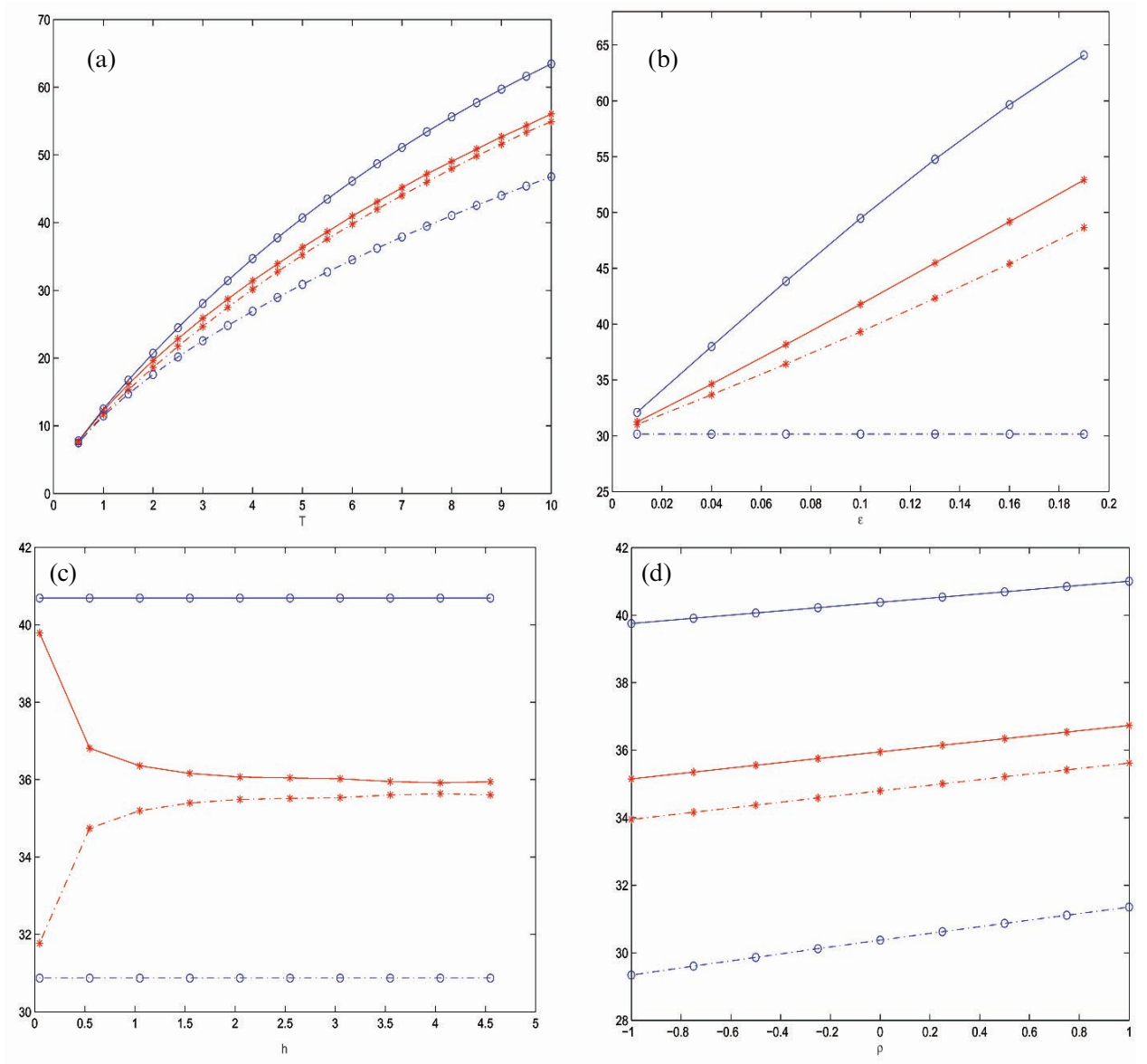
Finally, in Figure 2 the credit spreads (Equation (26)) are plotted as a function of some model parameters (the promised principal $F$, the correlation coefficient $\rho$, the gap $\epsilon$ and the intensity $h$ ), together with the corresponding values obtained under the Merton model.

Figure 2 Credit spreads as a function of the parameters $F, \epsilon, h$ and $\rho$. For each plot the fixed parameters were chosen as $\kappa=1, \sigma^{(r)}=0.02, g_{2}=0.05, g_{1}=0.1, h=1, S_{0}=100$, $F=90, \sigma^{(s)}=0.2, \rho=0.5$ (for colours see online version)
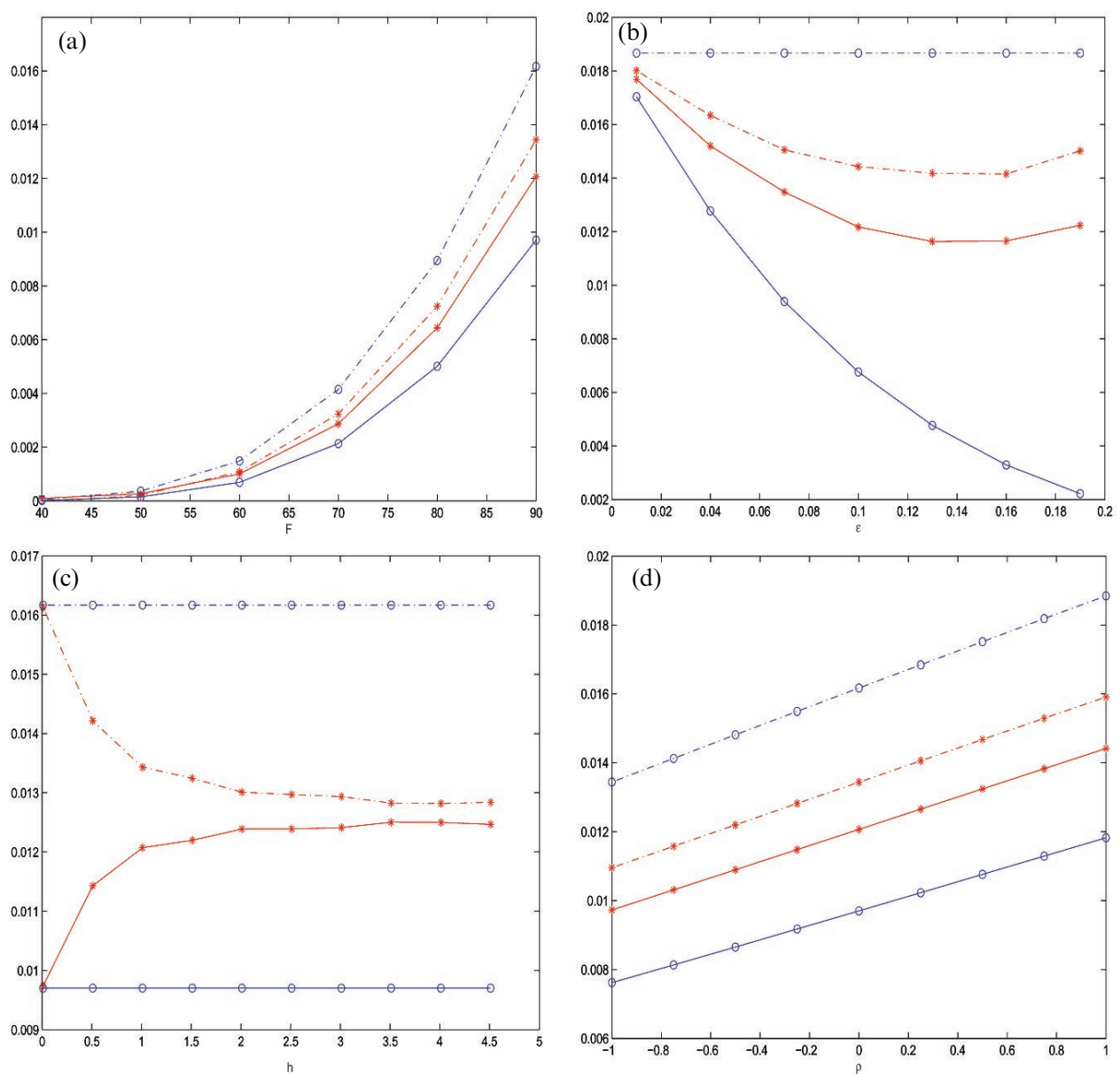

\section{References}

Abramowitz, M. and Stegun, A. (Eds) (1972) Handbook of Mathematical Functions, New York: Dover Publications Inc.

Ang, A. and Bekaert, G. (2002) 'Regime switches in interest rates', Journal of Business and Economic Statistics, Vol. 20, pp.163-182.

Bansal, R. and Zhou, H. (2002) 'Term structure of interest rates with regime shifts', Journal of Finance, Vol. 57, pp.1997-2043. 
Bjork, T. (2000) Arbitrage Theory in Continuous Time, Oxford.

Bjork, T., Kabanov, Y. and Runggaldier, W. (1997) 'Bond market in the presence of marked point processes', Mathematical Finance, Vol. 7, No. 2, pp.211-223.

Brémaud, P. (1981) Point Processes and Queues, Berlin: Springer; New York: Heidelberg.

Buffington, J. and Elliott, R.J. (2002) 'American options with regime switching', International Journal of Theoretical and Applied Finance, Vol. 5, pp.497-514.

Dai, Q. and Singleton, K. (2003) 'Term structure dynamics in theory and reality', The Review of Financial Studies, Vol. 16, No. 3, pp.631-678.

Dai, Q., Singleton, K. and Yang, W. (2005) 'Regime shifts in a dynamic term structure model of US treasury bond yields', Working Paper.

Duffie, D. and Singleton, K. (2003) Credit Risk. Pricing, Measurement and Management, Princeton Series in Finance.

Elliott, R.J., Chan, L. and Siu, T.K. (2005) 'Option pricing and Esscher transform under regime switching', Annals of Finance, Vol. 1, pp.423-432.

Geman, H., El Karoui, N. and Rochet, J.C. (1995) 'Changes of numéraire, changes of probability measure and option pricing', Journal of Applied Probability, Vol. 32, No. 2, pp.443-458.

Glasserman, P. and Merener, N. (2004) 'Convergence of a discretization scheme for jumpdiffusion processes with state-dependent intensities', Proceedings of the Royal Society, Vol. 460, No. 2041, pp.111-127.

Hamilton, J. (1989) 'A new approach to the economic analysis of non stationary time series and the business cycle', Econometrica, Vol. 57, pp.357-384.

Hansen, A.T. and Poulsen, R. (2000) 'A simple regime switching term structure model', Finance and Stochastics, Vol. 4, pp.409-429.

Jamshidian, F. (1989) 'An exact bond option formula', Journal of Finance, Vol. 44, pp.205-209.

Landen, C. (2000) 'Bond pricing in a hidden Markov model of the short rate', Finance and Stochastics, Vol. 4, pp.371-389.

Merton, D. (1973) 'The theory of rational option pricing', Bell Journal of Economics and Management Science, Vol. 4, pp.141-183.

Merton, D. (1974) 'On the pricing of corporate debt: the risk structure of interest rates', Journal of Finance, Vol. 29, pp.449-470.

Naik, V. and Lee, M.H. (1997) 'Yield curve dynamics with discrete shifts in economic regimes: theory and estimation', Working Paper, University of British Columbia.

Protter, P. (1990) Stochastic Integration and Differential Equations, Berlin: Springer.

Runggaldier, W.J. (2002) 'Jump-diffusion models', in Handbook of Heavy Tailed Distributions in Finance, S.T. Rachev (Ed.), Handbooks in Finance, Book 1, W. Ziemba Series Ed.), North-Holland: Elsevier, 2003, pp.169-209.

Shimko, D.C., Tejima, N. and Van Deventer, D. (1993) 'The pricing of risky debts when interest rates are stochastic', Journal of Fixed Income, Vol. 3, No. 2, pp.58-65.

Vasicek, O. (1977) 'An equilibrium characterization of the term structure', Journal of Financial Economics, Vol. 5, pp.177-188.

Wong, H.Y. and Kwok, Y.K. (2003) 'Jump diffusion models for risky debts: quality spread differentials', Int. J. Theor. and Appl. Finance, Vol. 6, pp.655-662.

$\mathrm{Wu}, \mathrm{S}$. and Zeng, Y. (2004) 'Affine regime-switching models for interest rate term structure', AMS Contemporary Mathematics, Mathematics of Finance, Vol. 351, pp.375-386. 